\title{
Asymptotic Analysis of Spectrum and Stability for One Class of Singularly Perturbed Neutral-Type Time-Delay Systems
}

\author{
Valery Y. Glizer
}

\author{
The Galilee Research Center for Applied Mathematics, ORT Braude College of Engineering, \\ Karmiel 2161002, Israel; valery48@braude.ac.il
}

\begin{abstract}
In this study, a singularly perturbed linear time-delay system of neutral type is considered. It is assumed that the delay is small of order of a small positive parameter multiplying a part of the derivatives in the system. This system is decomposed asymptotically into two much simpler parameter-free subsystems, the slow and fast ones. Using this decomposition, an asymptotic analysis of the spectrum of the considered system is carried out. Based on this spectrum analysis, parameterfree conditions guaranteeing the exponential stability of the original system for all sufficiently small values of the parameter are derived. Illustrative examples are presented.
\end{abstract}

Keywords: time-delay system of neutral type; singular perturbation; spectrum analysis; exponential stability

MSC: 34K06; 34K20; 34K26

check for

updates

Citation: Glizer, V.Y. Asymptotic Analysis of Spectrum and Stability for One Class of Singularly Perturbed Neutral-Type Time-Delay Systems. Axioms 2021, 10, 325. https:// doi.org/10.3390/axioms10040325

Academic Editor: Clemente Cesarano

Received: 20 October 2021

Accepted: 23 November 2021

Published: 30 November 2021

Publisher's Note: MDPI stays neutral with regard to jurisdictional claims in published maps and institutional affiliations.

Copyright: (C) 2021 by the author. Licensee MDPI, Basel, Switzerland. This article is an open access article distributed under the terms and conditions of the Creative Commons Attribution (CC BY) license (https:// creativecommons.org/licenses/by/ $4.0 /)$.

\section{Introduction}

Singularly perturbed differential systems, which can serve as adequate and convenient for analysis mathematical models of real-life multi-time-scale dynamical systems, are studied extensively in the literature (see e.g., [1-5] and references therein). One of the important classes of such systems from the theoretical and practical viewpoints is the class of time-delay systems. Brief surveys of results in this topic can be found in $[1,6]$.

Analysis of the spectrum of a linear time-invariant differential system (either undelayed or delayed), i.e., the analysis of the set of roots of its characteristic equation, is one of the basic approaches to the study of such a system. This analysis allows to derive the structure of the general solution of the system and many other quantitative and qualitative properties of its solutions (see e.g., $[2,3,7,8]$ ).

Since a singularly perturbed system depends on a small parameter $\varepsilon>0$, its characteristic equation also depends on this parameter. Using this feature of the characteristic equation, the structure of the set of its roots, valid for all sufficiently small $\varepsilon$, can be studied. Such a study can be carried out using a decomposition of the original singularly perturbed system into two much simpler $\varepsilon$-free subsystems, the slow and fast ones. This decomposition is called the slow-fast decomposition. In the literature, there are two known methods of such a decomposition, the exact and asymptotic ones. To the best of our knowledge, the exact slow-fast decomposition of singularly perturbed time-delay systems was developed only for the systems which are not of the neutral type. First, such a result was proposed in [9] where a singularly perturbed linear autonomous system with small delays both, point-wise and distributed, in the fast state variable was analyzed. A further extension of the exact slow-fast decomposition method was proposed in $[6,10]$ where singularly perturbed linear autonomous systems with point-wise and distributed small delays in both, slow and fast, state variables were studied. In [11], the exact slow-fast decomposition was proposed for a linear singularly perturbed time-invariant system having multiple point-wise commensurate non-small delays only in the slow state variable, while the fast state variable is delay free. In [12], a singularly perturbed linear time-invariant system with delay on time scales was considered. The delay 
in the form of Stieltjes integral appears only in the slow state variable. The exact slow-fast decomposition of this system was proposed in this work. For the asymptotic slow-fast decomposition of singularly perturbed time-delay differential systems of both, non neutral and neutral, types one can see e.g., [1,3,12-20] and references therein.

The structure of the spectrum of undelayed singularly perturbed differential systems was analyzed in a number of works in the literature (see e.g., [4,21-24]). Dependence on a parameter of the spectrum of time-delay differential systems, was also studied the literature. Thus, in [2], a regularly perturbed system was considered. Asymptotic behavior (with respect of the parameter) of its spectrum was studied in the case where the spectrum elements of the respective nominal (unperturbed) equation are simple. Preserving the asymptotic stability property for the spectrum of a regularly perturbed time-delay system was studied in [3]. Time-delay systems with commensurate delays, the spectrum of which has pure imaginary elements, were considered in [25-27]. A small perturbation was imposed on the delays. Conditions, guaranteeing that this perturbation shifts the pure imaginary elements of the spectrum to the left-hand side of the complex plane, were derived in these works. The asymptotic behavior of the spectrum of singularly perturbed time-delay differential systems, which are not of the neutral type, was also studied in the literature. Thus in [28], such a study was carried out for a differential-difference system with a small delay proportional to the positive small multiplier for a part of the derivatives. In [29,30], the asymptotic behavior of the spectrum was analyzed for singularly perturbed systems with the general type of the delay in the form of Stieltjes integral. In these papers, as in [28], the delay is assumed to be small. In [31], it was also considered a singularly perturbed system with the general type of the delay in the form of Stieltjes integral and the asymptotic behavior of its spectrum was studied. However, in contrast with $[29,30]$, the delay only in the fast state variable is small, while the delay in the slow state variable is non-small (in an order of 1). In the papers [28-31] the spectrum analysis is based on the asymptotic slow-fast decomposition of the considered systems. In [6] the spectrum of a singularly perturbed differential system with multiple point-wise and distributed small delays was analyzed using the exact slow-fast decomposition of the system. In [11], the spectrum analysis of a singularly perturbed linear time-invariant system with multiple point-wise commensurate non-small delays only in the slow state variable was carried out by using the exact slow-fast decomposition of this system. In [12], the spectrum of a singularly perturbed linear time-invariant system with the delay (in the form of Stieltjes integral) only in the slow state variable was analyzed based on the exact slow-fast decomposition of this system.

An important application of the spectrum analysis of a singularly perturbed system is the study of the exponential stability of this system. It should be noted that the exponential stability and the equivalent to it $L^{2}$-stability of singularly perturbed linear autonomous time-delay differential systems were studied in a number of works in the literature. Mainly, the case of non-neutral type systems was considered. Thus, in $[6,9,10]$, the stability study is based on the exact slow-fast decomposition of various such systems with the small delays either in the fast state variable or in both, slow and fast, state variables. In $[29,30]$, the study of the exponential stability for various singularly perturbed systems with the general type of the small delay in the form of Stieltjes integral is based on the asymptotic slow-fast decomposition of the original system. In [15], a singularly perturbed differential system with multiple point-wise and distributed state delays was considered. The delays in the slow state variable are non-small, while the delays in the fast state variable are small of order of the small parameter of singular perturbation $\varepsilon>0$. Based on the asymptotic slowfast decomposition of the considered system, as well as on its equivalent transformation to a system of integral equations, sufficient conditions for the exponential stability of this system were derived. In [18], a singularly perturbed linear time-invariant system with nonsmall point-wise delays is considered. Using the frequency domain method, $\varepsilon$-dependent and $\varepsilon$-independent sufficient conditions for the asymptotic stability of this system are obtained. In [32], the exponential stabilization of a singularly perturbed controlled linear autonomous system without delays by a linear state-feedback control with a non-small 
delay only in the slow state variable was analyzed using the state-space approach. A similar stabilization problem by a linear state-feedback control with both, small and non-small, state delays was studied in [33] using the frequency domain approach. The exponential stability and stabilization of a singularly perturbed system with the delay (in the form of Stieltjes integral) only in the slow state variable were studied in [12] by application of the exact slow-fast decomposition of this system. In [16,30,34-36], the exponential/asymptotic stability and stabilization problems for various singularly perturbed time-delay systems were studied in the framework of the Linear Matrix Inequalities method. In contrast to the above mentioned works, the stability of singularly perturbed time-delay systems of the neutral type was studied much less. To the best of our knowledge, there is only one paper [13] in the literature devoted to such a study. In this paper, a singularly perturbed linear time-invariant neutral type system with non-small point-wise delays is considered. Sufficient conditions, $\varepsilon$-dependent and $\varepsilon$-independent, for the asymptotic stability of this system are obtained in the framework of the frequency domain method.

In the present paper, we consider a singularly perturbed linear time-invariant timedelay differential system of neutral type. We deal with the case where the delay is small of order of a small positive multiplier $\varepsilon$ for a part of the derivatives in the system. To the best of our knowledge, such a type of singularly perturbed time-delay systems has not been considered yet in the literature. We analyze the spectrum of this system and its exponential stability. As mentioned above, the asymptotic behavior of the spectrum of singularly perturbed differential systems without delays, as well as of singularly perturbed time-delay systems of non-neutral type, has been studied extensively in the literature. However, to the best of our knowledge, the asymptotic behavior of the spectrum of a singularly perturbed time-delay differential system of the neutral type is analyzed for the first time in the literature in the present paper. To analyze this spectrum, the asymptotic decomposition of the corresponding characteristic equation in the form of an $\varepsilon$-dependent quasi-polynomial equation is carried out. This decomposition results in two much simpler parameter-independent equations: polynomial and quasi-polynomial ones. Using some proper assumptions on the structure of the sets' roots of these equations, the structure of the spectrum of the original singularly perturbed differential system, valid for all sufficiently small values of $\varepsilon$, is established. Based on this result, parameter-independent sufficient conditions for the exponential stability of the considered system are derived in the framework of the state-space method. These conditions, being $\varepsilon$-free, guarantee the exponential stability of the original singularly perturbed system for all sufficiently small values of $\varepsilon$.

The paper is organized as follows. In the next section, the problem is rigorously formulated. Based on this formulation, the objectives of the paper are rigorously stated. Some auxiliary results are presented in Section 3. The structure of the spectrum of the original singularly perturbed system is studied in Section 4. Stability analysis of this system is carried out in Section 5. Section 6 is devoted to illustrative examples. Conclusions are placed in Section 7.

The following main notations are applied in the paper:

(1) $E^{n}$ denotes the $n$-dimensional real Euclidean space, $\|\cdot\|$ denotes the norm in this space;

(2) $I_{n}$ denotes the $n$-dimensional identity matrix;

(3) $\operatorname{Re} \lambda$ and $\operatorname{Im} \lambda$ denote the real and imaginary parts, respectively, of a complex number $\lambda$;

(4) $\operatorname{col}(x, y)$, where $x \in E^{n}$ and $y \in E^{m}$, denotes a column block-vector with the upper block $x$ and the lower block $y$;

(5) $C\left[a, b ; E^{n}\right]$ is the space of continuous functions $f(t):[a, b] \rightarrow E^{n},\|\cdot\|_{C}$ denotes the uniform norm in $C\left[a, b ; E^{n}\right]$. 


\section{Problem Formulation}

\subsection{Original System}

The system under consideration is:

$$
\begin{aligned}
\frac{d}{d t}\left[x_{t}(0)+A_{1} x_{t}(-h)\right]= & B_{1} x_{t}(0)+B_{2} y_{t}(0)+H_{1} x_{t}(-h)+H_{2} y_{t}(-h) \\
& +\int_{-h}^{0}\left[G_{1}(\eta) x_{t}(\eta)+G_{2}(\eta) y_{t}(\eta)\right] d \eta, \quad t \geq 0, \\
\varepsilon \frac{d}{d t}\left[y_{t}(0)+A_{2} y_{t}(-h)\right]= & B_{3} x_{t}(0)+B_{4} y_{t}(0)+H_{3} x_{t}(-h)+H_{4} y_{t}(-h) \\
& +\int_{-h}^{0}\left[G_{3}(\eta) x_{t}(\eta)+G_{4}(\eta) y_{t}(\eta)\right] d \eta, \quad t \geq 0,
\end{aligned}
$$

where

$$
x_{t}(\eta) \triangleq x(t+\varepsilon \eta), \quad y_{t}(\eta) \triangleq y(t+\varepsilon \eta), \quad \eta \in[-h, 0] ;
$$

$x(t) \in E^{n}, y(t) \in E^{m} ; \varepsilon>0$ is a small parameter; $h>0$ is a given number independent of $\varepsilon ; A_{i}, B_{j}$ and $H_{j},(i=1,2 ; j=1, \ldots, 4)$ are given constant matrices of corresponding dimensions; $G_{j}(\eta),(j=1, \ldots, 4)$ are given piecewise continuous matrix-valued functions of corresponding dimensions for $\eta \in[-h, 0]$.

The system (1) and (2) is a singularly perturbed linear time-invariant functionaldifferential system of neutral type. It is infinite-dimensional with the state variables $(x(t), x(t+\varepsilon \eta))$ and $(y(t), y(t+\varepsilon \eta)), \eta \in[-h, 0)$. Equation (1) and the Euclidean part $x(t)$ of the state variable $(x(t), x(t+\varepsilon \eta))$ are called a slow mode and a slow Euclidean state variable of (1) and (2). Equation (2) and the state variable $(y(t), y(t+\varepsilon \eta))$ are called a fast mode and a fast state variable of (1) and (2).

For any $\varepsilon>0$ and $\eta \in[-h, 0]$, let us denote:

$$
\begin{gathered}
z_{t}(\eta) \triangleq \operatorname{col}\left(x_{t}(\eta), y_{t}(\eta)\right), \\
E_{\varepsilon} \triangleq\left(\begin{array}{cc}
I_{n} & 0 \\
0 & \varepsilon I_{m}
\end{array}\right), \quad A \triangleq\left(\begin{array}{cc}
A_{1} & 0 \\
0 & A_{2}
\end{array}\right), \quad B \triangleq\left(\begin{array}{ll}
B_{1} & B_{2} \\
B_{3} & B_{4}
\end{array}\right), \\
H \triangleq\left(\begin{array}{ll}
H_{1} & H_{2} \\
H_{3} & H_{4}
\end{array}\right), \quad G(\eta) \triangleq\left(\begin{array}{ll}
G_{1}(\eta) & G_{2}(\eta) \\
G_{3}(\eta) & G_{4}(\eta)
\end{array}\right) .
\end{gathered}
$$

Using these notations, we can rewrite the system (1) and (2) in the equivalent form as:

$$
E_{\varepsilon} \frac{d}{d t}\left[z_{t}(0)+A z_{t}(-h)\right]=B z_{t}(0)+H z_{t}(-h)+\int_{-h}^{0} G(\eta) z_{t}(\eta) d \eta, \quad t \geq 0
$$

Using the form (6) of the original system (1) and (2) and the results of [2], we obtain its characteristic equation (with respect to $\lambda$ ) as:

$$
\operatorname{det} \Delta(\lambda, \varepsilon)=0,
$$

where

$$
\begin{array}{r}
\Delta(\lambda, \varepsilon)=\lambda E_{\varepsilon}+\lambda \exp (-\lambda \varepsilon h) E_{\varepsilon} A-B \\
-\exp (-\lambda \varepsilon h) H-\int_{-h}^{0} \exp (\lambda \varepsilon \eta) G(\eta) d \eta .
\end{array}
$$

In what follows, we call (7) the original characteristic equation. 
For the sake of the spectrum analysis of the original system (1) and (2), we are going to decompose this system asymptotically into two much simpler $\varepsilon$-free subsystems (the slow and fast ones).

\subsection{Slow Subsystem}

To obtain this subsystem, we set formally $\varepsilon=0$ in (1) and (2). Thus, we have

$$
\begin{aligned}
A_{s} \frac{d x_{s}(t)}{d t} & =B_{1 s} x_{s}(t)+B_{2 s} y_{s}(t), & & t \geq 0, \\
0 & =B_{3 s} x_{s}(t)+B_{4 s} y_{s}(t), & & t \geq 0,
\end{aligned}
$$

where $x_{s}(t) \in E^{n}$ and $y_{s}(t) \in E^{m}$ are state variables;

$$
A_{s}=I_{n}+A_{1}, \quad B_{j s}=B_{j}+H_{j}+\int_{-h}^{0} G_{j}(\eta) d \eta, \quad j=1, \ldots, 4 .
$$

The slow subsystem (9) is a descriptor (differential-algebraic) system. Moreover, the first equation of this system is, in general, a singular differential equation. In addition, the slow subsystem is delay-free.

\section{If}

$$
\operatorname{det} A_{s} \neq 0 \text {, }
$$

and

$$
\operatorname{det} B_{4 s} \neq 0 \text {, }
$$

then the slow subsystem (9) can be reduced to the following regular differential equation with respect to $x_{s}$ :

$$
\frac{d x_{s}(t)}{d t}=A_{s}^{-1} B_{s} x_{s}(t), \quad t \geq 0
$$

where

$$
B_{s}=B_{1 s}-B_{2 s} B_{4 s}^{-1} B_{3 s} .
$$

The differential Equation (13) is also called the slow subsystem of the system (1) and (2). The characteristic equation with respect to $\lambda$ of the slow subsystem (13) is

$$
\operatorname{det} \Delta_{s}(\lambda)=0, \quad \Delta_{s}(\lambda)=\lambda I_{n}-A_{s}^{-1} B_{s} .
$$

In what follows, we call (15) the slow characteristic equation.

\subsection{Fast Subsystem}

The fast subsystem is formally derived from Equations (1) and (2) as follows. Firstly, the state variable $(x(t), x(t+\varepsilon \eta))$ is removed from (2), which yields the equation

$$
\varepsilon \frac{d}{d t}\left[y_{t}(0)+A_{2} y_{t}(-h)\right]=B_{4} y_{t}(0)+H_{4} y_{t}(-h)+\int_{-h}^{0} G_{4}(\eta) y_{t}(\eta) \eta, t \geq 0 .
$$

Secondly, the following transformations of the variables are made in the system consisting of (1) and (16):

$$
t=\varepsilon \xi
$$


$x(\varepsilon(\xi+\eta))=x_{f}(\xi+\eta) \triangleq x_{f, \xi}(\eta), \quad y(\varepsilon(\xi+\eta))=y_{f}(\xi+\eta) \triangleq y_{f, \xi}(\eta), \quad \eta \in[-h, 0]$,

where $\xi$, called the stretched time, is a new independent variable; $\left(x_{f}(\xi), x_{f}(\xi+\eta)\right)$ and $\left(y_{f}(\xi), y_{f}(\xi+\eta)\right), \eta \in[-h, 0)$, are new state variables.

Thus, we obtain the system

$$
\begin{gathered}
\frac{d}{d \xi}\left[x_{f, \xi}(0)+A_{1} x_{f, \xi}(-h)\right]=\varepsilon\left[B_{1} x_{f, \xi}(0)+B_{2} y_{f, \xi}(0)+H_{1} x_{f, \xi}(-h)\right. \\
\left.+H_{2} y_{f, \xi}(-h)+\int_{-h}^{0}\left[G_{1}(\eta) x_{f, \xi}(\eta)+G_{2}(\eta) y_{f, \xi}(\eta)\right] d \eta\right], \quad \xi \geq 0, \\
\frac{d}{d \xi}\left[y_{f, \xi}(0)+A_{2} y_{f, \xi}(-h)\right]=B_{4} y_{f, \xi}(0) \\
+H_{4} y_{f, \xi}(-h)+\int_{-h}^{0} G_{4}(\eta) y_{f, \xi}(\eta) d \eta, \quad \xi \geq 0 .
\end{gathered}
$$

Thirdly, we formally set $\varepsilon=0$ in the Equation (19), which yields

$$
\frac{d}{d \xi}\left[x_{f, \xi}(0)+A_{1} x_{f, \xi}(-h)\right]=0, \quad \xi \geq 0 .
$$

Finally, integrating Equation (21) from $\xi=0$ to any $\xi \geq 0$ with zero initial condition $x_{f}(\eta)=0, \eta \in[-h, 0]$, we obtain Equation

$$
x_{f, \xi}(0)+A_{1} x_{f, \xi}(-h)=0, \quad \xi \geq 0 .
$$

Equations (20) and (22) constitute the fast subsystem, associated with the original system (1) and (2). In this subsystem, Equation (20) is a neutral-type functional-differential equation with respect to $y_{f}(\cdot)$, while Equation (22) is a difference equation for $x_{f}(\cdot)$ with a continuous independent variable. Thus, Equations (20) and (22) are not connected to each other. Additionally, it should be noted that the stretched time $\xi$ is expressed by the original time $t$ in the form $\xi=t / \varepsilon$. Therefore, for any $t>0, \lim _{\varepsilon \rightarrow+0} \xi=+\infty$.

The characteristic equation with respect to $\mu$ of the fast subsystem has the form

$$
\operatorname{det} \Delta_{f}(\mu)=0 \text {, }
$$

where

$$
\begin{array}{r}
\Delta_{f}(\mu)=\left(\begin{array}{cc}
\Delta_{f, 1}(\mu) & 0 \\
0 & \Delta_{f, 2}(\mu)
\end{array}\right), \\
\Delta_{f, 1}(\mu)=I_{n}+\exp (-\mu h) A_{1}, \\
\Delta_{f, 2}(\mu)=\mu I_{m}+\mu \exp (-\mu h) A_{2}-B_{4} \\
-\exp (-\mu h) H_{4}-\int_{-h}^{0} \exp (-\mu \eta) G_{4}(\eta) d \eta .
\end{array}
$$

The quasi-polynomial Equation (23) can be rewritten equivalently as:

$$
\operatorname{det} \Delta_{f, 1}(\mu) \operatorname{det} \Delta_{f, 2}(\mu)=0 .
$$

In what follows, we call (23) (and, therefore, (25)) the fast characteristic equation.

Remark 1. Note that the equations

$$
\operatorname{det} \Delta_{f, 1}(\mu)=0,
$$


and

$$
\operatorname{det} \Delta_{f, 2}(\mu)=0
$$

are the characteristic equations of the difference Equation (22) and the functional-differential Equation (20), respectively.

Let $S_{f}$ be the set of all distinct roots of the fast characteristic Equation (23) (and, therefore, (25)). Let $S_{f, 1}$ and $S_{f, 2}$ be the sets of all distinct roots of the characteristic Equations (26) and (27), respectively. Then,

$$
S_{f}=S_{f, 1} \bigcup S_{f, 2} .
$$

Remark 2. Due to Equation (10) and the inequalities (11) and (12), Equations (26) and (27) do not have zero roots.

\subsection{Asymptotic Decomposition of the Original Characteristic Equation}

In this subsection we show that the $\varepsilon$-dependent original characteristic Equation (7) can be decomposed asymptotically into the $\varepsilon$-free slow (15) and fast (23) characteristic equations.

Let us start with the slow characteristic equation. Setting formally $\varepsilon=0$ in (7) and (8) and taking into account (10), we obtain

$$
\operatorname{det} \bar{\Delta}(\lambda)=0,
$$

where

$$
\bar{\Delta}(\lambda)=\Delta(\lambda, 0)=\lambda E_{0}\left(I_{n+m}+A\right)-\bar{B}, \quad \bar{B}=\left(\begin{array}{ll}
B_{1 s} & B_{2 s} \\
B_{3 s} & B_{4 s}
\end{array}\right),
$$

and $E_{0}=\left.E_{\varepsilon}\right|_{\varepsilon=0}$.

Using the block form of the matrix $A$ (see the notation (5)), as well as the block forms of the matrices $\bar{B}$ and $E_{0}$, we can rewrite the matrix $\bar{\Delta}(\lambda)$ in the block form as:

$$
\bar{\Delta}(\lambda)=\left(\begin{array}{cc}
\lambda A_{s}-B_{1 s} & -B_{2 s} \\
-B_{3 s} & -B_{4 s}
\end{array}\right),
$$

where $A_{s}$ is given in (10).

Applying the formula for the determinant of a block matrix (see [37]) to (31), and taking into account the inequality (12) and Equation (14), we obtain for any complex $\lambda$ :

$$
\operatorname{det} \bar{\Delta}(\lambda)=(-1)^{m} \operatorname{det}\left(\lambda A_{s}-B_{s}\right) \operatorname{det} B_{4 s} .
$$

Finally, using the inequality (11), we have

$$
\operatorname{det} \bar{\Delta}(\lambda)=(-1)^{m} \operatorname{det} A_{s} \operatorname{det}\left(\lambda I_{n}-A_{s}^{-1} B_{s}\right) \operatorname{det} B_{4 s} .
$$

Comparing (33) to (15) and taking into account the inequalities (11) and (12), we can observe the following. The set of roots of the slow characteristic Equation (15) and the set of roots of the polynomial with respect to $\lambda$ (33) coincide with each other. Furthermore, setting formally $\varepsilon=0$ in the original characteristic Equation (7) and dividing the resulting equation by $(-1)^{m} \operatorname{det} A_{s} \operatorname{det} B_{4 s}$, we obtain the slow characteristic Equation (15). 
Proceed to obtaining the fast characteristic equations from the original characteristic Equation (7). For this purpose, first, we rewrite (7) in the equivalent form

$$
\begin{gathered}
\operatorname{det} \hat{\Delta}_{1}(\lambda, \varepsilon)=0, \\
\hat{\Delta}_{1}(\lambda, \varepsilon)=\varepsilon\left(E_{\varepsilon}\right)^{-1} \Delta(\lambda, \varepsilon)=\varepsilon \lambda I_{n+m}+\varepsilon \lambda \exp (-\lambda \varepsilon h) A-\varepsilon\left(E_{\varepsilon}\right)^{-1} B \\
-\exp (-\lambda \varepsilon h) \varepsilon\left(E_{\varepsilon}\right)^{-1} H-\int_{-h}^{0} \exp (\lambda \varepsilon \eta) \varepsilon\left(E_{\varepsilon}\right)^{-1} G(\eta) d \eta .
\end{gathered}
$$

By the transformation of the variable $\lambda=\mu / \varepsilon$, where $\mu$ is a new variable, Equation (34) becomes

$$
\begin{gathered}
\operatorname{det} \hat{\Delta}_{2}(\mu, \varepsilon)=0 \\
\hat{\Delta}_{2}(\mu, \varepsilon)=\mu I_{n+m}+\mu \exp (-\mu h) A-\varepsilon\left(E_{\varepsilon}\right)^{-1} B \\
-\exp (-\mu h) \varepsilon\left(E_{\varepsilon}\right)^{-1} H-\int_{-h}^{0} \exp (\mu \eta) \varepsilon\left(E_{\varepsilon}\right)^{-1} G(\eta) d \eta .
\end{gathered}
$$

Remark 3. It should be noted the following. If, for a given $\varepsilon>0, \lambda$ is a root of the original characteristic Equation (7), then $\mu=\varepsilon \lambda$ is a root of Equation (35). Vice versa: if, for a given $\varepsilon>0$, $\mu$ is a root of Equation (35), then $\lambda=\mu / \varepsilon$ is a root of the original characteristic Equation (7).

Remark 4. Note that the transformation of the variable $\lambda=\mu / \varepsilon$ in (34) corresponds to the transformation of the independent variable $t=\varepsilon \xi$ in (1) and (2).

Now, setting formally $\varepsilon=0$ in (35), we obtain

$$
\begin{gathered}
\operatorname{det} \widetilde{\Delta}(\mu)=0, \\
\widetilde{\Delta}(\mu)=\mu I_{n+m}+\mu \exp (-\mu h) A-\mathcal{E} B \\
-\exp (-\mu h) \mathcal{E} H-\int_{-h}^{0} \exp (\mu \eta) \mathcal{E} G(\eta) d \eta,
\end{gathered}
$$

where the $(n+m) \times(n+m)$-matrix $\mathcal{E}$ has the form

$$
\mathcal{E}=\left(\begin{array}{ll}
0 & 0 \\
0 & I_{m}
\end{array}\right)
$$

Using the block forms of the matrices $A, B, H, G(\eta)$ (see the notation (5)), we can represent the matrix $\widetilde{\Delta}(\mu)$ in the block form as:

$$
\widetilde{\Delta}(\mu)=\left(\begin{array}{cc}
\mu \Delta_{f, 1}(\mu) & 0 \\
-B_{3}-\exp (-\mu h) H_{3}-\int_{-h}^{0} \exp (\mu \eta) G_{3}(\eta) d \eta & \Delta_{f, 2}(\mu)
\end{array}\right),
$$

where $\Delta_{f, 1}(\mu)$ and $\Delta_{f, 2}(\mu)$ are given in (24).

Due to (37), the quasi-polynomial equation $\operatorname{det} \widetilde{\Delta}(\mu)=0$ in (36) can be rewritten as:

$$
\mu^{n} \operatorname{det} \Delta_{f, 1}(\mu) \operatorname{det} \Delta_{f, 2}(\mu)=0 .
$$

Comparing (38) to (25) (and, therefore, to (23)), and using the inequalities (11), (12) and Remark 2 yield that the set of all roots of the fast characteristic Equation (25) coincides with the set of all nonzero roots of the Equation (38). Moreover, the fast characteristic Equation (25) (and, therefore, (23)) can be obtained from the original characteristic Equation (7) in the following way: (i) to transform (7) into (34); (ii) to transform the variable $\lambda=\mu / \varepsilon$ in (34), which yields (35); (iii) to set formally $\varepsilon=0$ in (35), which yields (36); (iv) to divide the quasi-polynomial equation in (36) (and, therefore, in (38)) by $\mu^{n}$. 


\subsection{Objectives of the Paper}

The objectives of the paper are:

(I) Based on the presumed structures of the roots' sets of the slow (15) and fast (25) characteristic equations to study a structure of the roots' set of the original characteristic Equation (7), valid for all sufficiently small values of $\varepsilon>0$;

(II) Based on this structure to analyze a stability of the original singularly perturbed system (1) and (2) and to estimate the solution of an initial-value problem for this system.

\section{Auxiliary Results}

\subsection{Properties of Roots of Some Quasi-Polynomial Equations}

Let $\lambda_{s, p},\left(p=1, \ldots, q_{s} \leq n\right)$ be all distinct roots of the slow characteristic Equation (15), i.e., all distinct eigenvalues of the matrix $A_{s}^{-1} B_{s}$. Remember that $A_{s}$ and $B_{s}$ are given in Equations (10) and (14), respectively.

Proceed to analysis of the set $S_{f, 1}$ of all distinct roots of Equation (26). In this analysis, the following two cases should be distinguished: $\left(a_{1}\right)$ all the eigenvalues of the matrix $A_{1}$ equal zero; $\left(b_{1}\right)$ at least one of the eigenvalues of $A_{1}$ differs from zero. In the case $\left(a_{1}\right)$, Equation (26) does not have roots. Let us treat the case $\left(b_{1}\right)$.

Lemma 1. Let $v_{p},\left(p=1, \ldots, q_{1}\right),\left(1 \leq q_{1} \leq n\right)$ be all distinct non-zero eigenvalues of the matrix $A_{1}$. Then, the set $S_{f, 1}$ has the form

$$
S_{f, 1}=\left\{\mu=\mu_{p, l}=(1 / h) \ln \left|v_{p}\right|+i\left(\varphi_{p}+\pi l\right) / h, p=1, \ldots, q_{1}, l= \pm 1, \pm 3, \ldots\right\},
$$

where $i$ is the imaginary unit; the angles $\varphi_{p},\left(p=1, \ldots, q_{1}\right)$ are defined by the conditions

$$
\cos \varphi_{p}=\operatorname{Re} v_{p} /\left|v_{p}\right|, \quad \sin \varphi_{p}=\operatorname{Im} v_{p} /\left|v_{p}\right|, \quad 0 \leq \varphi_{p} \leq \pi, p=1, \ldots, q_{1} .
$$

Proof. First of all let us note that, due to the form of the matrix $\Delta_{f, 1}(\mu)$ (see Equation (24)), all distinct roots of Equation (26) coincide with all roots of the following equation with respect to $\mu$ :

$$
\prod_{p=1}^{q_{1}}\left(1+\exp (-\mu h) v_{p}\right)=0
$$

Thus, a complex number $\mu$ is a root of this equation if and only if it is a root of one of the following equations:

$$
1+\exp (-\mu h) v_{p}=0, \quad p=1, \ldots, q_{1},
$$

which directly yields the statement of the lemma.

Consider the following quasi-polynomial equation with respect to $\mu$ :

$$
\operatorname{det} \Psi_{f}(\mu)=0, \quad \Psi_{f}(\mu)=I_{m}+\exp (-\mu h) A_{2} .
$$

Note that (41) is the characteristic equation of the following difference equation with the continuous independent variable:

$$
\widetilde{y}_{\xi}(0)+A_{2} \widetilde{y}_{\xi}(-h)=0, \quad \xi \geq 0,
$$

where $\widetilde{y}_{\xi}(\eta) \triangleq \widetilde{y}(\xi+\eta), \eta \in[-h, 0]$; for any $\xi \geq-h, \widetilde{y}(\xi) \in E^{m}$.

For Equation (41), similarly to Equation (26), we can distinguish the following cases: $\left(a_{2}\right)$ all the eigenvalues of the matrix $A_{2}$ equal zero; $\left(b_{2}\right)$ at least one of the eigenvalues of $A_{2}$ differs from zero. In the case $\left(a_{2}\right)$, Equation (41) does not have roots. In the case $\left(b_{2}\right)$, we have the following assertion. 
Lemma 2. Let $\kappa_{p},\left(p=1, \ldots, q_{2}\right),\left(1 \leq q_{2} \leq m\right)$ be all distinct non-zero eigenvalues of the matrix $A_{2}$. Then, the set $\Omega_{f}$ of all distinct roots of Equation (41) has the form

$$
\Omega_{f}=\left\{\mu=\mu_{p, l}=(1 / h) \ln \left|\kappa_{p}\right|+i\left(\phi_{p}+\pi l\right) / h, p=1, \ldots, q_{2}, l= \pm 1, \pm 3, \ldots\right\},
$$

where $i$ is the imaginary unit; the angles $\phi_{p},\left(p=1, \ldots, q_{2}\right)$ are defined by the conditions

$$
\cos \phi_{p}=\operatorname{Re} \kappa_{p} /\left|\kappa_{p}\right|, \quad \sin \phi_{p}=\operatorname{Im} \kappa_{p} /\left|\kappa_{p}\right|, \quad 0 \leq \phi_{p} \leq \pi, \quad p=1, \ldots, q_{2} .
$$

Proof. The lemma is proved quite similarly to Lemma 1.

Let us denote

$$
\beta \triangleq \sup _{\mu \in S_{f, 2}} \operatorname{Re} \mu, \quad \gamma_{1} \triangleq \max _{p=\overline{1, q_{1}}}(1 / h) \ln \left|\nu_{p}\right|, \quad \gamma_{2} \triangleq \max _{p=\overline{1, q_{2}}}(1 / h) \ln \left|\kappa_{p}\right| .
$$

Remember that $S_{f, 2}$ is the set of all distinct roots of the quasi-polynomial Equation (27) which is the characteristic equation of the functional-differential Equation (20).

Remark 5. If all the eigenvalues of the matrix $A_{1}$ equal zero, we set $\gamma_{1}=-\infty$. Similarly, if all the eigenvalues of the matrix $A_{2}$ equal zero, we set $\gamma_{2}=-\infty$.

Due to the results of [2], the value $\beta$ is finite, i.e.,

$$
\beta<+\infty
$$

Let

$$
\alpha \triangleq \max \left\{\beta, \gamma_{1}, \gamma_{2}\right\}
$$

Lemma 3. Let the inequalities (11) and (12) be satisfied. Let $\left\{\varepsilon_{k}\right\}$ and $\left\{\mu_{k}\right\},(k=1,2, \ldots)$ be any sequences such that:

(i) $\varepsilon_{k}>0,(k=1,2, \ldots)$;

(ii) $\lim _{k \rightarrow+\infty} \varepsilon_{k}=0$;

(iii) there exists a number $\delta>0$ such that $\operatorname{Re} \mu_{k} \geq \alpha+\delta,(k=1,2, \ldots)$;

(iv) each pair $\left(\mu_{k}, \varepsilon_{k}\right),(k=1,2, \ldots)$ satisfies the original characteristic equation in the form (35), i.e., det $\hat{\Delta}_{2}\left(\mu_{k}, \varepsilon_{k}\right)=0$.

Then, there exists a subsequence of the sequence $\left\{\mu_{k}\right\}$, which converges to zero.

Proof. First, let us show that the sequence $\left\{\mu_{k}\right\}$ is bounded. Assume the opposite, i.e., $\left\{\mu_{k}\right\}$ is unbounded. In this case, there exists a subsequence of $\left\{\mu_{k}\right\}$ which tends to infinity in the complex plane. For the sake of simplicity (but without loss of generality), we can assume that this subsequence coincides with the sequence $\left\{\mu_{k}\right\}$. Thus, $\lim _{k \rightarrow+\infty}\left|\mu_{k}\right|=+\infty$. In particular, this means that $\mu_{k} \neq 0$ for all sufficiently large $k$. Using this observation, as well as the block forms of the matrices $E_{\varepsilon}, A, B, H$ and $G(\eta)$, we can rewrite the equality $\operatorname{det} \hat{\Delta}\left(\mu_{k}, \varepsilon_{k}\right)=0$ in the following form for all sufficiently large $k$ :

$$
\operatorname{det} \hat{\Delta}_{3}\left(\mu_{k}, \varepsilon_{k}\right)=0 \text {, }
$$


where

$$
\begin{array}{r}
\hat{\Delta}_{3}\left(\mu_{k}, \varepsilon_{k}\right)=\left(\begin{array}{cc}
\hat{\Delta}_{3,1}\left(\mu_{k}, \varepsilon_{k}\right) & \hat{\Delta}_{3,2}\left(\mu_{k}, \varepsilon_{k}\right) \\
\hat{\Delta}_{3,3}\left(\mu_{k}, \varepsilon_{k}\right) & \hat{\Delta}_{3,4}\left(\mu_{k}, \varepsilon_{k}\right)
\end{array}\right), \\
\hat{\Delta}_{3,1}\left(\mu_{k}, \varepsilon_{k}\right)=\Delta_{f, 1}\left(\mu_{k}\right)-\left(\varepsilon_{k} / \mu_{k}\right)\left[B_{1}+\exp \left(-\mu_{k} h\right) H_{1}\right. \\
\left.+\int_{-h}^{0} \exp \left(\mu_{k} \eta\right) G_{1}(\eta) d \eta\right], \\
\hat{\Delta}_{3,2}\left(\mu_{k}, \varepsilon_{k}\right)=-\left(\varepsilon_{k} / \mu_{k}\right)\left[B_{2}+\exp \left(-\mu_{k} h\right) H_{2}\right. \\
\left.+\int_{-h}^{0} \exp \left(\mu_{k} \eta\right) G_{2}(\eta) d \eta\right], \\
\hat{\Delta}_{3,3}\left(\mu_{k}, \varepsilon_{k}\right)=-\left(1 / \mu_{k}\right)\left[B_{3}+\exp \left(-\mu_{k} h\right) H_{3}\right. \\
\left.+\int_{-h}^{0} \exp \left(\mu_{k} \eta\right) G_{3}(\eta) d \eta\right], \\
\hat{\Delta}_{3,4}\left(\mu_{k}, \varepsilon_{k}\right)=\Psi_{f}\left(\mu_{k}\right)-\left(1 / \mu_{k}\right)\left[B_{4}+\exp \left(-\mu_{k} h\right) H_{4}\right. \\
\left.+\int_{-h}^{0} \exp \left(\mu_{k} \eta\right) G_{4}(\eta) d \eta\right] .
\end{array}
$$

Remember that $\Delta_{f, 1}(\mu)$ and $\Psi_{f}(\mu)$ are given in (24) and (41), respectively.

Due to the condition (iii) of the lemma, the sequence $\left\{\exp \left(-\mu_{k} h\right)\right\}$ is bounded and the sequence $\left\{\exp \left(\mu_{k} \eta\right)\right\}$ is bounded uniformly in $\eta \in[-h, 0]$. Moreover, due to this condition, Lemmas 1 and 2, and Remark 5, we directly have that

$$
\left|\operatorname{det} \Delta_{f, 1}\left(\mu_{k}\right)\right| \geq \chi, \quad\left|\operatorname{det} \Psi_{f}\left(\mu_{k}\right)\right| \geq \chi, \quad k=1,2, \ldots,
$$

where $\chi>0$ is some number independent of $k$.

In addition, let us note that $\lim _{k \rightarrow+\infty}\left(\varepsilon_{k} / \mu_{k}\right)=0$ and $\lim _{k \rightarrow+\infty}\left(1 / \mu_{k}\right)=0$. Taking into account these observations and using Equation (49), we can represent the equality (48) in the following form for all sufficiently large $k$ :

$$
\operatorname{det} \Delta_{f, 1}\left(\mu_{k}\right) \operatorname{det} \Psi_{f}\left(\mu_{k}\right)+f\left(\mu_{k}, \varepsilon_{k}\right)=0,
$$

where $f\left(\mu_{k}, \varepsilon_{k}\right)$ is some function of $\mu_{k}$ and $\varepsilon_{k}$, satisfying the condition $\lim _{k \rightarrow+\infty} f\left(\mu_{k}, \varepsilon_{k}\right)=$ 0 . The latter, along with the inequalities (50), means that the equality (51) is contradictive. Therefore, the sequence $\left\{\mu_{k}\right\}$ cannot be unbounded.

Since the sequence $\left\{\mu_{k}\right\}$ is bounded, then its convergent subsequence exists. For the sake of simplicity (but without loss of generality), we assume that this subsequence coincides with $\left\{\mu_{k}\right\}$. Let us denote $\mu_{\lim } \triangleq \lim _{k \rightarrow+\infty} \mu_{k}$. Due to the condition (iii) of the lemma,

$$
\operatorname{Re} \mu_{\lim } \geq \alpha+\delta .
$$

Now, calculating the limit of the equality $\operatorname{det} \hat{\Delta}_{2}\left(\mu_{k}, \varepsilon_{k}\right)=0$ for $k \rightarrow+\infty$, and taking into account Equations (35)-(38), we directly obtain the equality

$$
\left(\mu_{\lim }\right)^{n} \operatorname{det} \Delta_{f, 1}\left(\mu_{\lim }\right) \operatorname{det} \Delta_{f, 2}\left(\mu_{\lim }\right)=0 .
$$

However, due to the inequality (52) and the definition of the number $\alpha$ (see Equations (45) and (47), the inequality (46) and Remark 5), we have that $\operatorname{det} \Delta_{f, 1}\left(\mu_{\text {lim }}\right) \neq 0$ and $\operatorname{det} \Delta_{f, 2}\left(\mu_{\text {lim }}\right) \neq 0$. Therefore, $\mu_{\lim }=0$, which completes the proof of the lemma.

As a direct consequence of Lemma 3, we have this assertion. 
Corollary 1. Let all the assumptions of Lemma 3 be valid. Then, the following inequality is satisfied: $\alpha<0$.

Lemma 4. Let the inequalities (11) and (12) be satisfied. Let $\left\{\varepsilon_{k}\right\}$ and $\left\{\lambda_{k}\right\},(k=1,2, \ldots)$ be any sequences such that:

(i) $\varepsilon_{k}>0,(k=1,2, \ldots)$;

(ii) $\lim _{k \rightarrow+\infty} \varepsilon_{k}=0$;

(iii) $\lim _{k \rightarrow+\infty} \varepsilon_{k} \lambda_{k}=0$;

(iv) each pair $\left(\lambda_{k}, \varepsilon_{k}\right),(k=1,2, \ldots)$ satisfies the original characteristic Equation (7).

Then, there exists a subsequence of the sequence $\left\{\lambda_{k}\right\}$, which converges to one of the numbers $\lambda_{s, p},\left(p=1, \ldots, q_{s}\right)$.

Proof. Taking into account the inequalities (11) and (12), the lemma is proved quite similarly to Lemma 2 and Corollary 2 of [28].

\subsection{Exponential Stability of the Difference Equations}

Let us start with Equation (22). For this equation, we assume that at least one of the eigenvalues of the matrix $A_{1}$ differs from zero, and we consider the initial condition

$$
x_{f}(\eta)=\psi_{x}(\eta), \quad \eta \in[-h, 0]
$$

where $\psi_{x}(\eta) \in C\left[-h, 0 ; E^{n}\right]$ is a given vector-valued function satisfying the equality

$$
\psi_{x}(0)+A_{1} \psi_{x}(-h)=0
$$

Definition 1. Equation (22) is called exponentially stable if, for any function $\psi_{x}(\eta) \in C\left[-h, 0 ; E^{n}\right]$ with the above mentioned property, the unique solution $x_{f}(\xi), \xi \geq 0$ of the initial-value problem (22) and (53) satisfies the inequality

$$
\left\|x_{f}(\xi)\right\| \leq c_{x} \exp \left(-\omega_{x} \xi\right)\left\|\psi_{x}(\eta)\right\|_{c}, \quad \xi \geq 0,
$$

where $c_{x}>0$ and $\omega_{x}>0$ are some constants independent of $\psi_{x}(\eta)$.

Lemma 5. Equation (22) is exponentially stable if and only if $\left|v_{p}\right|<1,\left(p=1, \ldots, q_{1}\right),(1 \leq$ $\left.q_{1} \leq n\right)$, where $v_{p},\left(p=1, \ldots, q_{1}\right)$ are all distinct non-zero eigenvalues of the matrix $A_{1}$.

Proof. The statement of the lemma directly follows from Lemma 1 and the results of the work [2].

Proceed to Equation (42). For this equation, similarly to the Equation (22), we assume that at least one of the eigenvalues of the matrix $A_{2}$ differs from zero, and we consider the initial condition

$$
\widetilde{y}(\eta)=\widetilde{\psi}_{y}(\eta), \quad \eta \in[-h, 0]
$$

where $\widetilde{\psi}_{y}(\eta) \in C\left[-h, 0 ; E^{m}\right]$ is a given vector-valued function satisfying the equality

$$
\widetilde{\psi}_{y}(0)+A_{2} \widetilde{\psi}_{y}(-h)=0 .
$$

Definition 2. Equation (42) is called exponentially stable if, for any function $\widetilde{\psi}_{y}(\eta) \in C\left[-h, 0 ; E^{m}\right]$ with the above mentioned property, the unique solution $\widetilde{y}(\xi), \xi \geq 0$ of the initial-value problem (42), (54) satisfies the inequality

$$
\|\widetilde{y}(\tilde{\xi})\| \leq \widetilde{c}_{y} \exp \left(-\widetilde{\omega}_{y} \xi\right)\left\|\widetilde{\psi}_{y}(\eta)\right\|_{C}, \quad \xi \geq 0,
$$

where $\widetilde{c}_{y}>0$ and $\widetilde{\omega}_{y}>0$ are some constants independent of $\widetilde{\psi}_{y}(\eta)$.

The following assertion directly follows from Lemma 2 and the results of the work [2]. 
Lemma 6. Equation (42) is exponentially stable if and only if $\left|\kappa_{p}\right|<1,\left(p=1, \ldots, q_{2}\right),(1 \leq$ $\left.q_{2} \leq m\right)$, where $\kappa_{p},\left(p=1, \ldots, q_{2}\right)$ are all distinct non-zero eigenvalues of the matrix $A_{2}$.

3.3. Exponential Stability of the Auxiliary Differential Equation and Neutral Type Functional-Differential Equation

We start with Equation (13), for which we immediately have the following assertion.

Lemma 7. Let the inequalities (11) and (12) be valid. Then, for any given vector $x_{s, 0} \in E^{n}$, the unique solution $x_{s}(t), t \geq 0$ of Equation (13) subject to the initial condition $x_{s}(0)=x_{s, 0}$ satisfies the inequality

$$
\begin{gathered}
\left\|x_{s}(t)\right\| \leq c_{s} \exp \left(-\omega_{s} t\right)\left\|x_{s, 0}\right\|, \\
c_{s}>0 \text { and } \omega_{s}>0 \text { are some constants independent of } x_{s, 0},
\end{gathered}
$$

if and only if all distinct roots $\lambda_{s, p},\left(p=1, \ldots, q_{s} \leq n\right)$ of the slow characteristic Equation (15) satisfy the inequalities $\operatorname{Re} \lambda_{s, p}<0,\left(p=1, \ldots, q_{s} \leq n\right)$.

Note that the inequality (55) means the exponential stability of Equation (13).

Proceed to Equation (20). For this equation, we consider the initial condition

$$
y_{f}(\eta)=\psi_{y}(\eta), \quad \eta \in[-h, 0]
$$

where $\psi_{y}(\eta) \in C\left[-h, 0 ; E^{m}\right]$ is a given vector-valued function.

Definition 3. Equation (20) is called exponentially stable if, for any function $\psi_{y}(\eta) \in C\left[-h, 0 ; E^{m}\right]$, the unique solution $y(\xi), \xi \geq 0$ of the initial-value problem (20), (56) satisfies the inequality

$$
\|y(\xi)\| \leq c_{y} \exp \left(-\omega_{y} \xi\right)\left\|\psi_{y}(\eta)\right\|_{C}, \quad \xi \geq 0,
$$

where $c_{y}>0$ and $\omega_{y}>0$ are some constants independent of $\psi_{y}(\eta)$.

By virtue of the results of the work [2], we immediately have the following assertion.

Lemma 8. Equation (20) is exponentially stable if and only if $\beta<0$, where the value $\beta$ is defined in Equation (45).

\section{Structure of the Roots' Set of the Original Characteristic Equation}

Let

$$
\begin{aligned}
\sigma_{\min } \triangleq \min _{p=1, q_{s}} \operatorname{Re} \lambda_{s, p}, \quad \sigma_{\max } \triangleq \max _{p=1, q_{s}} \operatorname{Re} \lambda_{s, p}, \\
\rho_{\min } \triangleq \min _{p=\overline{1, q_{s}}} \operatorname{Im} \lambda_{s, p}, \quad \rho_{\max } \triangleq \max _{p=\overline{1, q_{s}}} \operatorname{Im} \lambda_{s, p} .
\end{aligned}
$$

Let $\delta>0$ be any given number. Consider the domain

$$
\mathcal{D}_{s}=\left\{\lambda: \operatorname{Re} \lambda \in\left(\sigma_{\min }-\delta, \sigma_{\max }+\delta\right), \operatorname{Im} \lambda \in\left(\rho_{\min }-\delta, \rho_{\max }+\delta\right)\right\},
$$

and, for a given $\varepsilon>0$, the domain

$$
\mathcal{D}_{f}(\varepsilon)=\{\lambda: \operatorname{Re} \lambda<(\alpha+\delta) / \varepsilon\}
$$

Remember, that the number $\alpha$ is defined by Equations (45) and (47), the inequality (46) and Remark 5.

Denote

$$
\mathcal{D}(\varepsilon) \triangleq \mathcal{D}_{s} \bigcup \mathcal{D}_{f}(\varepsilon)
$$


Theorem 1. Let the inequalities (11) and (12) be satisfied. Then, there exists a number $\varepsilon^{*}=$ $\varepsilon^{*}(\delta)>0$ such that, for all $\varepsilon \in\left(0, \varepsilon^{*}\right]$, each root $\lambda(\varepsilon)$ of the original characteristic Equation (7) belongs to the domain $\mathcal{D}(\varepsilon)$.

Proof. We prove the theorem by contradiction, i.e., we assume that the statement of the theorem is wrong. This assumption yields the existence of two sequences $\left\{\varepsilon_{k}\right\}$ and $\left\{\lambda_{k}\right\}$ satisfying the following conditions:

(a) $\varepsilon_{k}>0,(k=1,2, \ldots)$;

(b) $\lim _{k \rightarrow+\infty} \varepsilon_{k}=0$;

(c) $\lambda_{k}$ does not belong to $\mathcal{D}_{f}\left(\varepsilon_{k}\right)$ for all $k \in\{1,2, \ldots\}$;

(d) $\lambda_{k}$ does not belong to $\mathcal{D}_{s}$ for all $k \in\{1,2, \ldots\}$;

(e) each pair $\left(\lambda_{k}, \varepsilon_{k}\right),(k=1,2, \ldots)$ satisfies the original characteristic Equation (7), i.e., $\operatorname{det} \Delta\left(\lambda_{k}, \varepsilon_{k}\right)=0$.

Consider the sequence $\left\{\mu_{k}\right\}$, where $\mu_{k}=\varepsilon_{k} \lambda_{k},(k=1,2, \ldots)$. Due to the condition (c) on the sequence $\left\{\lambda_{k}\right\}$ and the definition of the domain $\mathcal{D}_{f}(\varepsilon)$ (see the Equation (59)), we have that $\operatorname{Re} \mu_{k} \geq \alpha+\delta,(k=1,2, \ldots)$. The latter, along with the conditions (a), (b) on the sequence $\left\{\varepsilon_{k}\right\}$ and Remark 3, means the fulfillment of all the conditions of Lemma 3. Thus, there exists a subsequence of $\left\{\mu_{k}\right\}$, which converges to zero. For the sake of simplicity (but without loss of generality), we can assume that this subsequence coincides with the sequence $\left\{\mu_{k}\right\}$. Since $\mu_{k}=\varepsilon_{k} \lambda_{k},(k=1,2, \ldots)$, then the sequences $\left\{\varepsilon_{k}\right\}$ and $\left\{\lambda_{k}\right\}$ satisfy the conditions of Lemma 4 . By virtue of this lemma, there exist a subsequence of $\left\{\lambda_{k}\right\}$ converging to one of the numbers $\lambda_{s, p},\left(p=1, \ldots, q_{s}\right)$. The latter, along with the Equations (57) and (58), means that infinitely many elements of the sequence $\left\{\lambda_{k}\right\}$ belong to the domain $\mathcal{D}_{s}$, which contradicts the condition (d) on the sequence $\left\{\lambda_{k}\right\}$. This contradiction proves the theorem.

\section{Stability Analysis of the Original Singularly Perturbed System}

In what follows, we assume:

$$
\alpha<0, \quad \sigma_{\max }<0 .
$$

Remember that the number $\alpha$ is defined by Equations (45) and (47), the inequality (46) and Remark 5; the number $\sigma_{\max }$ is defined in the Equation (57).

Remark 6. Subject to the first inequality in (61), we have the following. For any given number $0<\delta<-\alpha$, there exists a number $\varepsilon_{1}^{*}>0$ such that, for all $\varepsilon \in\left(0, \varepsilon_{1}^{*}\right], \mathcal{D}_{s} \cap \mathcal{D}_{f}(\varepsilon)=\varnothing$. This relation between $\mathcal{D}_{s}$ and $\mathcal{D}_{f}(\varepsilon)$ yields the separation of the roots of the original characteristic Equation (7). Namely, the roots belonging to the domain $\mathcal{D}_{s}$ constitute the set $\mathcal{R}_{s}(\varepsilon)$ of the so called slow roots, while the roots belonging to the domain $\mathcal{D}_{f}(\varepsilon)$ constitute the set $\mathcal{R}_{f}(\varepsilon)$ of the so called fast roots of (7).

Remark 7. If $\alpha<0$, then $\gamma_{1}<0$. Therefore, $\left|v_{p}\right|<1,\left(p=1, \ldots, q_{1}\right)$. The latter means, that the inequality (11) is satisfied.

As a direct consequence of Theorem 1 and Remarks 6 and 7, we have the following assertion.

Corollary 2. Let the inequalities (12) and (61) be satisfied. Then, there exists a number $\varepsilon^{0}>0$ such that for all $\varepsilon \in\left(0, \varepsilon^{0}\right]$ the following inequalities are valid

$$
\begin{aligned}
& \sup _{\lambda(\varepsilon) \in \mathcal{R}_{s}(\varepsilon)} \operatorname{Re} \lambda(\varepsilon) \leq \sigma_{\max }<0, \sup _{\lambda(\varepsilon) \in \mathcal{R}_{f}(\varepsilon)} \operatorname{Re} \lambda(\varepsilon) \leq \alpha / \varepsilon<0, \\
& \sup _{\lambda(\varepsilon) \in \mathcal{R}_{s}(\varepsilon) \cup \mathcal{R}_{f}(\varepsilon)} \operatorname{Re} \lambda(\varepsilon) \leq \sigma_{\max }<0 .
\end{aligned}
$$


For a given $\varepsilon>0$, let us consider the initial conditions for the system (1) and (2)

$$
x(\tau)=\theta_{x}(\tau), \quad y(\tau)=\theta_{y}(\tau), \quad \tau \in[-\varepsilon h, 0],
$$

where $\theta_{x}(\tau) \in C\left[-\varepsilon h, 0 ; E^{n}\right]$ and $\theta_{y}(\tau) \in C\left[-\varepsilon h, 0 ; E^{m}\right]$ are any given functions.

Theorem 2. Let the inequalities (12) and (61) be satisfied. Then, for any number $\varepsilon \in\left(0, \varepsilon^{0}\right]$, any number $\sigma \in\left(\sigma_{\max }, 0\right)$ independent of $\varepsilon$, and any functions $\theta_{x}(\tau) \in C\left[-\varepsilon h, 0 ; E^{n}\right]$ and $\theta_{y}(\tau) \in C\left[-\varepsilon h, 0 ; E^{m}\right]$, there exists a number $c(\sigma)>0$, independent of the number $\varepsilon$ and the functions $\theta_{x}(\tau), \theta_{y}(\tau)$, such that the unique solution $(x(t, \varepsilon), y(t, \varepsilon)), t \in[0,+\infty)$ of the initialvalue problem (1), (2) and (63) satisfies the inequalities

$$
\begin{aligned}
& \|x(t, \varepsilon)\| \leq c(\sigma) \exp (\sigma t)\left\|\theta_{x}(\tau)\right\|_{C}, \quad t \in[0,+\infty), \\
& \|y(t, \varepsilon)\| \leq c(\sigma) \exp (\sigma t)\left\|\theta_{y}(\tau)\right\|_{C}, \quad t \in[0,+\infty) .
\end{aligned}
$$

Proof. Let $\varepsilon \in\left(0, \varepsilon^{0}\right]$ be any given. In the original system (1) and (2), let us make the transformation of the variables (17) and (18). Due to this transformation, we obtain a new system, equivalent to (1) and (2). This new system consists of Equation (19) and the equation

$$
\begin{aligned}
& \frac{d}{d \xi}\left[y_{f, \xi}(0)+A_{2} y_{f, \xi}(-h)\right]=B_{3} x_{f, \xi}(0)+B_{4} y_{f, \xi}(0)+H_{3} x_{f, \xi}(-h) \\
& \quad+H_{4} y_{f, \xi}(-h)+\int_{-h}^{0}\left[G_{3}(\eta) x_{f, \xi}(\eta)+G_{4}(\eta) y_{f, \xi}(\eta)\right] d \eta, \quad \xi \geq 0 .
\end{aligned}
$$

For the system (19) and (65), let us consider the initial conditions

$$
x_{f}(\zeta)=\theta_{x, f}(\zeta), \quad y_{f}(\zeta)=\theta_{y, f}(\zeta), \quad \zeta \in[-h, 0],
$$

where

$$
\theta_{x, f}(\zeta)=\theta_{x}(\varepsilon \zeta), \quad \theta_{y, f}(\zeta)=\theta_{y}(\varepsilon \zeta), \quad \zeta \in[-h, 0] .
$$

Since $\theta_{x}(\tau) \in C\left[-\varepsilon h, 0 ; E^{n}\right]$ and $\theta_{y}(\tau) \in C\left[-\varepsilon h, 0 ; E^{m}\right]$, then $\theta_{x, f}(\zeta) \in C\left[-h, 0 ; E^{n}\right]$ and $\theta_{y, f}(\zeta) \in C\left[-h, 0 ; E^{m}\right]$.

By virtue of Remarks 3 and 4, Equation (35) is the characteristic equation of the system (19) and (16). Moreover, for any $\varepsilon \in\left(0, \varepsilon^{0}\right]$, a complex number $\mu$ is a root of (35) if and only if the number $\lambda=\mu / \varepsilon$ is a root of Equation (7). Due to this observation and the third inequality in (62), we directly have that any root $\mu(\varepsilon), \varepsilon \in\left(0, \varepsilon^{0}\right]$ of Equation (35) satisfies the inequality

$$
\operatorname{Re} \mu(\varepsilon) \leq \varepsilon \sigma_{\max }, \quad \varepsilon \in\left(0, \varepsilon^{0}\right] .
$$

In addition, let us note that all the coefficients of the system (19) and (65) are uniformly bounded for $\varepsilon \in\left[0, \varepsilon^{0}\right]$. The latter, along with the inequality (68) and the results of the work [2], directly yields the existence of a number $c(\sigma)>0$, independent of the number $\varepsilon$ and the functions $\theta_{x, f}(\zeta), \theta_{y, f}(\zeta)$, such that the unique solution $\left(x_{f, \xi}(\xi, \varepsilon), y_{f, \xi}(\xi, \varepsilon)\right)$, $\xi \in[0,+\infty)$ of the initial-value problem (19), (65) and (66) satisfies the inequalities

$$
\begin{array}{ll}
\left\|x_{f, \xi}(\xi, \varepsilon)\right\| \leq c(\sigma) \exp (\varepsilon \sigma \xi)\left\|\theta_{x, f}(\zeta)\right\|_{C}, & \xi \in[0,+\infty), \\
\left\|y_{f, \xi}(\xi, \varepsilon)\right\| \leq c(\sigma) \exp (\varepsilon \sigma \xi)\left\|\theta_{y, f}(\zeta)\right\|_{c}, & \xi \in[0,+\infty) .
\end{array}
$$

Using Equation (67), and the equivalence of the system (1) and (2) and the system (19) and (65), we obtain that the unique solution $(x(t, \varepsilon), y(t, \varepsilon)), t \in[0,+\infty)$ of the initialvalue problem (1), (2) and (63) can be represented in the form $\left(x_{f, \xi}(t / \varepsilon, \varepsilon), y_{f, \xi}(t / \varepsilon, \varepsilon)\right)$, $t \in[0,+\infty)$. Therefore, by the transformation of the independent variables $\xi=t / \varepsilon$, $t \in[0,+\infty)$ and $\zeta=\tau / \varepsilon, \tau \in[-\varepsilon h, 0]$, the inequalities (69) become the inequalities (64). The latter, along with the assumption that $\varepsilon$ is any given number from the interval $\left(0, \varepsilon^{0}\right]$, proves the statement of the theorem. 
Remark 8. The statement of Theorem 2 means that the original system (1) and (2) is exponentially stable with the coefficient for the exponent and the decay constant independent of $\varepsilon>0$ for all sufficiently small values of this parameter. Thus, the exponential stability of the original system (1) and (2) is robust with respect to $\varepsilon$ for all such values.

Corollary 3. Let in the original system (1) and (2), the matrices $A_{1}$ and $A_{2}$ have non-zero eigenvalues. Let the inequality (12) be satisfied. Let the difference Equations (22) and (42) be exponentially stable. Let the differential Equation (13) and the neutral type functional-differential Equation (20) be exponentially stable. Then, the original system (1) and (2) is exponentially stable robustly with respect to $\varepsilon>0$ for all sufficiently small values of this parameter.

Proof. From Lemmas 5-8, and from the definitions of the number $\alpha$ (see the Equations (45) and (47) and the inequality (46)) and the number $\sigma_{\max }$ (see the Equation (57)), we immediately have the fulfillment of the inequalities in (61). Now, the statement of the corollary is a direct consequence of Theorem 2 and Remark 8.

\section{Examples}

In this section two examples are presented. These examples are nontrivial and they allow us to clearly illustrate the theoretical results of the paper, avoiding too complicated analytical and numerical calculations.

\subsection{Example 1}

Consider a particular case of system (1) and (2) with the following data:

$$
\begin{array}{r}
A_{1}=\left(\begin{array}{cc}
1 & -0.25 \\
3 & 0
\end{array}\right), B_{1}=\left(\begin{array}{cc}
-2 & 1 \\
4 & -3
\end{array}\right), B_{2}=\left(\begin{array}{c}
5 \\
-1
\end{array}\right), H_{1}=\left(\begin{array}{cc}
-1 & 6 \\
-5 & 4
\end{array}\right), \\
H_{2}=\left(\begin{array}{c}
2 \\
-1
\end{array}\right), G_{1}(\eta)=\left(\begin{array}{c}
2 \eta \\
3(\eta+1)^{2}-2 \eta
\end{array}\right), \\
G_{2}(\eta)=\left(\begin{array}{c}
4 \eta^{3} \\
-4(\eta+1)^{3}
\end{array}\right), A_{2}=0.5, \quad B_{3}=(2,-2), \quad B_{4}=-6, \\
H_{3}=(3,4), H_{4}=-3, G_{3}(\eta)=\left(-\frac{\pi}{2} \sin \left(\frac{\pi}{2} \eta\right), 5(\eta+1)^{4}\right), G_{4}(\eta) \equiv 0 .
\end{array}
$$

In this example, the eigenvalues of the matrix $A_{1}$ are $v_{1}=(1+\sqrt{2} i) / 2, v_{2}=(1-$ $\sqrt{2} i) / 2$, where $i$ is the imaginary unit. Thus,

$$
\left|v_{p}\right|=\sqrt{3} / 2<1, \quad p=1,2 .
$$

Since in this example, the matrix $A_{2}$ becomes scalar value, its single eigenvalue $\kappa_{1}$ coincides with this value, i.e., $\kappa_{1}=A_{2}=0.5$. Thus,

$$
\left|\kappa_{1}\right|=0.5<1 .
$$

Proceed to the calculation of the matrices in the Equation (10). We have

$$
\begin{array}{r}
A_{s}=\left(\begin{array}{cc}
2 & -0.25 \\
3 & 1
\end{array}\right), \quad B_{1 s}=\left(\begin{array}{cc}
-4 & 8 \\
0 & 2
\end{array}\right), \quad B_{2 s}=\left(\begin{array}{c}
6 \\
-3
\end{array}\right) \\
B_{3 s}=(6,3), \quad B_{4 s}=-9 .
\end{array}
$$

Thus, the inequality (12) is satisfied, and the matrix $B_{S}$ (see the Equation (14)) becomes as:

$$
B_{s}=\left(\begin{array}{cc}
0 & 10 \\
-2 & 1
\end{array}\right)
$$


Now, using (13), (73) and (74), we obtain the slow subsystem of system (1) and (2) with the data (70)

$$
\frac{d x_{s}(t)}{d t}=\frac{1}{11}\left(\begin{array}{ll}
-2 & 41 \\
-16 & -112
\end{array}\right) x_{s}(t), \quad t \geq 0, \quad x_{s}(t) \in E^{2} .
$$

The eigenvalues of the matrix of the coefficients in system (75) are $\lambda_{s, 1}=-0.7571$, $\lambda_{s, 2}=-9.6066$. Thus, by virtue of (57), $\sigma_{\max }=-0.7571$ meaning

$$
\sigma_{\max }<0
$$

Proceed to the deriving the fast subsystem, associated with the original system of this example (1), (2) and (70). Using (20) and (22), we obtain

$$
\begin{gathered}
\frac{d}{d \xi}\left[y_{f, \xi}(0)+0.5 y_{f, \xi}(-1)\right]=-6 y_{f, \xi}(0)-3 y_{f, \xi}(-1), \quad \xi \geq 0, \\
x_{f, \xi}(0)+\left(\begin{array}{cc}
1 & -0.25 \\
3 & 0
\end{array}\right) x_{f, \xi}(-1)=0, \quad \xi \geq 0,
\end{gathered}
$$

where for all $\xi \geq-1, y_{f}(\xi)$ is a scalar, while $x_{f}(\xi) \in E^{2}$.

Along with Equations (77) and (78), let us write down Equation (42) in this example. Due to the data (70), this equation becomes the following scalar equation:

$$
\widetilde{y}_{\xi}(0)+0.5 \widetilde{y}_{\xi}(-1)=0, \quad \xi \geq 0 .
$$

Now, let us check whether the conditions of Corollary 3 are fulfilled for the original system of this example (1), (2) and (70). The eigenvalues of the matrix $A_{1}$ and the scalar value $A_{2}$ differ from zero. Moreover, as mentioned above, the inequality (12) is satisfied. Further, due to the inequalities in (71) and Lemma 5, the difference Equation (78) is exponentially stable. Similarly, due to the inequality (72) and Lemma 6, the difference Equation (79) is exponentially stable. Using the inequalities (71) and (76) and Lemma 7, we directly obtain the exponential stability of the differential Equation (75). Thus, to apply Corollary 3 to the stability analysis of the system (1), (2) and (70), we should show the exponential stability of the neutral type functional-differential Equation (77). Due to Lemma 8 and Equations (24), (27), (45) and (70), Equation (77) is exponentially stable if and only if the following inequality is satisfied:

$$
\sup _{\mu \in S_{f, 2}} \operatorname{Re} \mu<0,
$$

where $S_{f, 2}$ is the set of all distinct roots of the quasi-polynomial equation

$$
\mu+0.5 \mu \exp (-\mu)+6+3 \exp (-\mu)=0 .
$$

Equation (81) has infinitely many roots, which are

$$
\begin{gathered}
\mu_{1}=-6, \quad \mu_{2}=-\ln 2+\pi i, \quad \mu_{3}=-\ln 2-\pi i, \quad \mu_{4}=-\ln 2+3 \pi i, \\
\mu_{5}=-\ln 2-3 \pi i, \quad \mu_{6}=-\ln 2+5 \pi i, \quad \mu_{7}=-\ln 2-5 \pi i, \ldots,
\end{gathered}
$$

where $i$ is the imaginary unit.

Thus, the inequality (80) is satisfied. Therefore, all the conditions of Corollary 3 are fulfilled for the original system of this example (1), (2) and (70), meaning that this system is exponentially stable robustly with respect to the parameter $\varepsilon>0$ for all its sufficiently small values. 


\subsection{Example 2}

Consider a particular case of the system (1) and (2) with the following data:

$$
\begin{array}{r}
A_{1}=\left(\begin{array}{cc}
-1 & 3 \\
-0.2 & 1
\end{array}\right), B_{1}=\left(\begin{array}{cc}
-1 & 1 \\
2 & 4
\end{array}\right), B_{2}=\left(\begin{array}{c}
-4 \\
1
\end{array}\right), H_{1}=\left(\begin{array}{cc}
2 & 5 \\
4 & 3
\end{array}\right), \\
H_{2}=\left(\begin{array}{c}
-1 \\
2
\end{array}\right), \quad G_{1}(\eta)=\left(\begin{array}{cc}
2(\eta+1) & -6(\eta+1)^{2} \\
-9 \eta^{2} & 4 \eta
\end{array}\right), \\
G_{2}(\eta)=\left(\begin{array}{c}
5 \eta^{4} \\
-5(\eta+1)^{4}
\end{array}\right), A_{2}=0.5723, \quad B_{3}=(-1,4), \quad B_{4}=-6, \\
H_{3}=(2,1), H_{4}=2, G_{3}(\eta)=\left(6 \eta^{5},-7 \eta^{6}\right), G_{4}(\eta) \equiv 0 .
\end{array}
$$

Calculating the eigenvalues of the matrix $A_{1}$, given in (83), we obtain $v_{1}=\sqrt{0.4}$, $v_{2}=-\sqrt{0.4}$. Thus,

$$
\left|v_{p}\right|=\sqrt{0.4}<1, \quad p=1,2 .
$$

In the present example, the matrix $A_{2}$ becomes scalar value, its single eigenvalue $\kappa_{1}$ coincides with this value, i.e., $\kappa_{1}=A_{2}=0.5723$. Thus,

$$
\left|\kappa_{1}\right|=0.5723<1 .
$$

Calculating the matrices in Equation (10), we obtain

$$
\begin{array}{r}
A_{s}=\left(\begin{array}{cc}
0 & 3 \\
-0.2 & 1
\end{array}\right), \quad B_{1 s}=\left(\begin{array}{ll}
2 & 4 \\
3 & 5
\end{array}\right), \quad B_{2 s}=\left(\begin{array}{c}
-4 \\
2
\end{array}\right) \\
B_{3 s}=(0,4), \quad B_{4 s}=-4
\end{array}
$$

Since $B_{4 s} \neq 0$, the inequality (12) is satisfied. Using Equation (14), we calculate the matrix $B_{S}$ as:

$$
B_{s}=\left(\begin{array}{ll}
2 & 0 \\
3 & 7
\end{array}\right) .
$$

Now, using Equations (13), (86) and (87), we obtain the slow subsystem of the system (1) and (2) with the data (83)

$$
\frac{d x_{s}(t)}{d t}=\frac{5}{3}\left(\begin{array}{cc}
-7 & -21 \\
0.4 & 0
\end{array}\right) x_{s}(t), \quad t \geq 0, \quad x_{s}(t) \in E^{2} .
$$

The spectrum of the system (88) consists of two elements $\lambda_{s, 1}=-9.1036$ and $\lambda_{s, 2}=-2.5631$. Thus, by virtue of (57), $\sigma_{\max }=-2.5631$ meaning

$$
\sigma_{\max }<0 .
$$

Proceed to the obtaining the fast subsystem, associated with the original system of this example (1), (2) and (83). Using Equations (20) and (22), we have

$$
\begin{gathered}
\frac{d}{d \xi}\left[y_{f, \xi}(0)+0.5723 y_{f, \xi}(-1)\right]=-6 y_{f, \xi}(0)+2 y_{f, \xi}(-1), \quad \xi \geq 0, \\
x_{f, \xi}(0)+\left(\begin{array}{cc}
-1 & 3 \\
-0.2 & 1
\end{array}\right) x_{f, \xi}(-1)=0, \quad \xi \geq 0,
\end{gathered}
$$

where for all $\xi \geq-1, y_{f}(\xi)$ is a scalar, while $x_{f}(\xi) \in E^{2}$. 
Along with Equations (90) and (91), let us write down Equation (42) in this example. Due to the data (83), this equation becomes the following scalar equation:

$$
\widetilde{y}_{\xi}(0)+0.5723 \widetilde{y}_{\xi}(-1)=0, \quad \xi \geq 0 .
$$

Now, we are going to check whether the conditions of Corollary 3 are fulfilled for the original system of this example (1), (2) and (83). The eigenvalues of the matrix $A_{1}$ and the scalar value $A_{2}$ differ from zero. The inequality (12) is satisfied as well. Due to the inequalities in (84) and Lemma 5, the difference Equation (91) is exponentially stable. Similarly, due to the inequality (85) and Lemma 6, the difference Equation (92) is exponentially stable. Inequalities (71), (76) and Lemma 7 directly yield the exponential stability of the differential Equation (88). Thus, to apply Corollary 3 to the stability analysis of the system (1), (2) and (83), we should show the exponential stability of the neutral type functional-differential Equation (90). Due to Lemma 8 and Equations (24), (27), (45) and (83), Equation (90) is exponentially stable if and only if the following inequality is satisfied:

$$
\sup _{\mu \in S_{f, 2}} \operatorname{Re} \mu<0,
$$

where $S_{f, 2}$ is the set of all distinct roots of the quasi-polynomial equation

$$
\mu+0.5723 \mu \exp (-\mu)+6-2 \exp (-\mu)=0 .
$$

It is verified directly that Equation (94) has the root $\mu=-0.6794-\frac{3 \pi}{2} i$, where $i$ is the imaginary unit. This root has a negative real part. To find other roots of this equation is rather a complicated task. Let us show that Equation (94) satisfies the inequality (93). First, let us show that this equation does not have roots with non-negative real parts. Note, that for any root $\mu$ of (94), the following inequality is satisfied:

$$
1+0.5723 \exp (-\mu) \neq 0 .
$$

Indeed, if there exists a root $\mu$ of (94) such that $1+0.5723 \exp (-\mu)=0$, then $6-2 \exp (-\mu)=0$, which yields the contradiction $-1 / 0.5723=3$. Therefore, the inequality (95) is correct for all roots $\mu$ of Equation (94). Using this inequality, we can rewrite this equation in the equivalent form

$$
\mu=\frac{2 \exp (-\mu)-6}{1+0.5723 \exp (-\mu)},
$$

which yields

$$
\operatorname{Re} \mu=\operatorname{Re}\left(\frac{2 \exp (-\mu)-6}{1+0.5723 \exp (-\mu)}\right) .
$$

This equation can be rewritten in the form

$$
\operatorname{Re} \mu=\frac{F_{\text {num }}(\mu)}{F_{\text {den }}(\mu)},
$$

where

$$
F_{\text {num }}(\mu)=-6-1.4338 \exp (-\operatorname{Re} \mu) \cos (\operatorname{Im} \mu)+1.1446 \exp (-2 \operatorname{Re} \mu),
$$

$$
F_{\operatorname{den}}(\mu)=[1+0.5723 \exp (-\operatorname{Re} \mu) \cos (\operatorname{Im} \mu)]^{2}+(0.5723)^{2} \exp (-2 \operatorname{Re} \mu) \sin ^{2}(\operatorname{Im} \mu) .
$$

Let us assume that Equation (96) (and, therefore, Equation (94)) has a root $\bar{\mu}$ with a non-negative real part, i.e., $\operatorname{Re} \bar{\mu} \geq 0$. Using this inequality and Equation (98), we directly 
have $F_{\text {num }}(\bar{\mu}) \leq-3.4216<0$. The latter, along with Equation (97) and the inequality $F_{\operatorname{den}}(\bar{\mu})>0$, contradicts the above assumed inequality $\operatorname{Re} \bar{\mu} \geq 0$. This contradiction proves that the real parts of all roots of Equation (96) (and, therefore, of the Equation (94)) are negative. Now, let us assume that there exists a sequence of these roots $\left\{\bar{\mu}_{k}\right\}_{k=1}^{+\infty}$ such that $\lim _{k \rightarrow+\infty} \operatorname{Re} \bar{\mu}_{k}=0$. Due to Equation (97), we have

$$
\operatorname{Re} \bar{\mu}_{k}=\frac{F_{\text {num }}\left(\bar{\mu}_{k}\right)}{F_{\text {den }}\left(\bar{\mu}_{k}\right)}, \quad k=1,2, \ldots
$$

Since $\operatorname{Re} \bar{\mu}_{k}$ tends to zero for $k \rightarrow+\infty$, then for all sufficiently large $k$,

$$
\begin{aligned}
& \left|F_{\text {num }}\left(\bar{\mu}_{k}\right)\right| \geq 6-1.4338 \exp \left(-\operatorname{Re} \bar{\mu}_{k}\right)-1.1446 \exp \left(-2 \operatorname{Re} \bar{\mu}_{k}\right)>0, \\
& 0<F_{\operatorname{den}}\left(\bar{\mu}_{k}\right) \leq 1+1.1446 \exp \left(-\operatorname{Re} \bar{\mu}_{k}\right)+(0.5723)^{2} \exp \left(-2 \operatorname{Re} \bar{\mu}_{k}\right) .
\end{aligned}
$$

Equation (100), along with the inequalities (101) and (102), yields for all sufficiently large $k$

$$
\left|\operatorname{Re} \bar{\mu}_{k}\right| \geq \frac{6-1.4338 \exp \left(-\operatorname{Re} \bar{\mu}_{k}\right)-1.1446 \exp \left(-2 \operatorname{Re} \bar{\mu}_{k}\right)}{1+1.1446 \exp \left(-\operatorname{Re} \bar{\mu}_{k}\right)+(0.5723)^{2} \exp \left(-2 \operatorname{Re} \bar{\mu}_{k}\right)}
$$

Calculating the limit of this inequality for $k \rightarrow+\infty$, we obtain the contradiction $0 \geq 1$.384. This contradiction proves that a sequence of roots $\left\{\bar{\mu}_{k}\right\}_{k=1}^{+\infty}$ of Equation (96) does not exist (and, therefore, of the Equation (94)) such that $\lim _{k \rightarrow+\infty} \operatorname{Re} \bar{\mu}_{k}=0$. This feature of the roots, along with the negativeness of real parts for all roots of Equation (94) shown above, immediately proves the inequality (93). Thus, we have shown the fulfillment of all the conditions of Corollary 3 for the original system of this example (1), (2) and (83), which means that this system is exponentially stable robustly with respect to the parameter $\varepsilon>0$ for all its sufficiently small values.

\section{Conclusions}

The singularly perturbed linear autonomous neutral type differential system, having point-wise and distributed delays, was considered in this paper. The case where the delays are proportional to the parameter of singular perturbation $\varepsilon>0$ has been investigated. This system significantly differs from the singularly perturbed systems studied in the literature. To the best of our knowledge, such a type of singularly perturbed systems has not been considered yet in the literature. It has required a considerably novel method of analysis, which has been elaborated in this paper. The asymptotic behavior of the spectrum (the set of all roots of the characteristic equation) of the considered system was studied. This study is based on the asymptotic decomposition of the original singularly perturbed system into two much simpler $\varepsilon$-free subsystems-the slow and fast ones. The slow subsystem is a lower Euclidean dimension (than the original system) un-delayed differential equation. The fast subsystem consists of two modes, which are not connected with each other. Moreover, both modes are of lower Euclidean dimensions than the original system. One of these modes is a neutral-type functional-differential equation, while the other mode is a difference equation with a continuous independent variable. Such a decomposition of the original singularly perturbed system, where the fast subsystem consists of two modes, is a considerably novel result in the field of singularly perturbed problems. The characteristic equations of the slow and fast subsystems, called the slow and fast characteristic equations, are also independent of $\varepsilon$. Moreover, these equations are considerably simpler than the characteristic equation for the original singularly perturbed system. The asymptotic decomposition of the original characteristic equation has been carried out, and the connection of this decomposition with the slow and fast characteristic equations has been established. Like the fast subsystem, the fast characteristic equation consists of two modes. This result 
also is significantly new. Based on the presumed structure of the spectrums of the slow and fast subsystems, the structure of the spectrum of the original singularly perturbed system has been derived. Although the assumptions on the structure of the spectrums of the slow and fast subsystems are $\varepsilon$-free, the obtained structure of the spectrum of the original singularly perturbed system is valid for all sufficiently small values of $\varepsilon$. Thus, the spectrum analysis of the rather complicated $\varepsilon$-dependent characteristic equation of the original singularly perturbed system has been reduced to the analysis of much simpler $\varepsilon$-free characteristic equations of the slow and fast subsystems. This result on the structure of the spectrum of the original singularly perturbed system has been applied to the study of its exponential stability. Namely, it has established the following. If the slow and fast subsystems are exponentially stable, and one more $\varepsilon$-free auxiliary difference equation with a continuous independent variable is exponentially stable, then the original singularly perturbed system is exponentially stable for all sufficiently small values of the parameter of singular perturbation $\varepsilon>0$. This result reduces the stability analysis of the complicated $\varepsilon$-dependent system to the analysis of several much simpler $\varepsilon$-free systems.

The future issues of this topic, which are interesting issues for future investigations, are the following: (a) the asymptotic analysis of the spectrum structure and the stability for the singularly perturbed linear autonomous neutral type differential system with small delays in the case where the spectrum of the functional-differential mode of the fast subsystem has pure imaginary elements, meaning that this mode is not exponentially stable; (b) the asymptotic analysis of the spectrum structure and the stability for the singularly perturbed linear autonomous neutral type differential system with the non-small (of order of 1 ) delays in the slow state variable $x(\cdot)$ and the small delays in the fast state variable $y(\cdot)$; (c) asymptotic solution of the initial-value and boundary-value problems for a singularly perturbed linear/nonlinear autonomous/nonautonomous neutral type differential system with small/non-small delays

Funding: This research received no external funding.

Institutional Review Board Statement: Not applicable.

Informed Consent Statement: Not applicable.

Data Availability Statement: Not applicable.

Conflicts of Interest: The authors declare no conflict of interest.

\section{References}

1. Glizer, V.Y. Controllability of Singularly Perturbed Linear Time Delay Systems; Birkhauser: Cham, Switzerland, 2021.

2. Hale, J.K.; Verduyn Lunel, S.M. Introduction to Functional Differential Equations; Springer: New York, NY, USA, 1993.

3. Halanay, A. Differential Equations: Stability, Oscillations, Time Lags; Academic Press: New York, NY, USA, 1966.

4. Kokotovic, P.V.; Khalil, H.K.; O’Reilly, J. Singular Perturbation Methods in Control: Analysis and Design; Academic Press: London, UK, 1986.

5. Vasil'eva, A.B.; Butuzov, V.F.; Kalachev, L.V. The Boundary Function Method for Singular Perturbation Problems; SIAM: Philadelphia, PA, USA, 1995.

6. Glizer, V.Y.; Fridman, E.; Feigin, Y. A novel approach to exact slow-fast decomposition of linear singularly perturbed systems with small delays. SIAM J. Control Optim. 2017, 55, 236-274. [CrossRef]

7. Hartman, P. Ordinary Differential Equations; SIAM: Philadelphia, PA, USA, 2002.

8. Bellman, R.; Cooke, K.L. Differential-Difference Equations; Academic Press: New York, NY, USA, 1963.

9. Fridman, E. Decoupling transformation of singularly perturbed systems with small delays. Z. Angew. Math. Mech. 1996, 76, 201-204.

10. Glizer, V.Y.; Fridman, E. Ho control of linear singularly perturbed systems with small state delay. J. Math. Anal. Appl. 2000, 250, 49-85. [CrossRef]

11. Tsekhan, O. Complete controllability conditions for linear singularly-perturbed time-invariant systems with multiple delays via Chang-type transformation. Axioms 2019, 8, 71. [CrossRef]

12. Pawluszewicz, E.; Tsekhan, O. Stability and Stabilisability of the Singularly Perturbed System with Delay on Time Scales: A Decomposition Approach. Internat. J. Control. 2021. [CrossRef] 
13. Chen, C.-F.; Pan, S.-T. Stability Analysis of Neutral Time-Delay Systems via Time-Scale Separation Technique, International Symposiums on Industrial Electronics, Mechatronics and Applications. 2007; pp. 63-70. Available online: https://www. researchgate.net/publication/268269311 (accessed on 26 March 2020).

14. Dmitriev, M.G.; Kurina, G.A. Singular perturbations in control problems. Autom. Remote Control 2006, 67, 1-43. [CrossRef]

15. Dragan, V.; Ionita, A. Exponential stability for singularly perturbed systems with state delays. In Proceedings of the 6th Colloquium on the Qualitative Theory of Differential Equations, Szeged, Hungary, 10-14 August 1999; pp. 1-8.

16. Fridman, E. Robust sampled-data $H_{\infty}$ control of linear singularly perturbed systems. IEEE Trans. Automat. Control 2006, 51, 470-475. [CrossRef]

17. Guglielmi, N.; Hairer, E. Asymptotic expansions for regularized state-dependent neutral delay equations. SIAM J. Math. Anal. 2012, 44, 2428-2458. [CrossRef]

18. Pan, S.-T.; Chen, C.-F.; Hsieh, J.-G. Stability analysis for a class of singularly perturbed systems with multiple time delays. J. Dyn. Syst. Meas. Control 2004, 126, 462-466. [CrossRef]

19. Wang, N. The asymptotic solution to singularly perturbed neutral differential difference equations. Ann. Differ. Equs. 2013, 1, 81-88.

20. Zhang, Y.; Naidu, D.S.; Cai, C.; Zou, Y. Singular perturbations and time scales in control theories and applications: An overview 2002-2012. Int. J. Inform. System Sci. 2014, 9, 1-36.

21. Luse, D.W. Frequency domain results for systems with multiple time scales. IEEE Trans. Automat. Control 1986, 31, 918-924. [CrossRef]

22. Luse, D.W.; Khalil, H.K. Frequency domain results for systems with slow and fast dynamics. IEEE Trans. Automat. Control 1985, 30, 1171-1179. [CrossRef]

23. Vishik, M.I.; Lyusternik, L.A. Regular degeneration and boundary layer for linear differential equations with small parameter. Uspekhi Mat. Nauk 1957, 12, 3-122. (In Russian)

24. Vishik, M.I.; Lyusternik, L.A. The solution of some perturbation problems for matrices and selfadjoint or non-selfadjoint differential equations I. Russ. Math. Surv. 1960, 15, 1-73. [CrossRef]

25. Chen, J.; Fu, P.; Niculescu, S.-I. Asymptotic behavior of imaginary zeros of linear systems with commensurate delays. In Proceedings of the 45th IEEE Conference on Decision and Control, San-Diego, CA, USA, 13-15 December 2006; pp. $1375-1380$.

26. Chen, J.; Fu, P.; Niculescu, S.-I. An eigenvalue perturbation stability analysis approach with applications to time-delay and polynomially dependent systems. In Proceedings of the 7th World Congress on Intelligent Control and Automation, Chongqing, China, 25-27 June 2008; pp. 307-312.

27. Fu, P.; Chen, J.; Niculescu, S.-I. High-order analysis of critical stability properties of linear time-delay systems. In Proceedings of the 2007 American Control Conference, New York, NY, USA, 9-13 July 2007; pp. 4921-4926.

28. Glizer, V.Y. Stabilizability and detectability of singularly perturbed linear time-invariant systems with delays in state and control. J. Dyn. Control Syst. 1999, 5, 153-172. [CrossRef]

29. Glizer, V.Y. Blockwise estimate of the fundamental matrix of linear singularly perturbed differential systems with small delay and its application to uniform asymptotic solution. J. Math. Anal. Appl. 2003, 278, 409-433.

30. Glizer, V.Y.; Fridman, E. Stability of singularly perturbed functional-differential systems: spectrum analysis and LMI approaches. IMA J. Math. Control Inform. 2012, 29, 79-111. [CrossRef]

31. Glizer, V.Y. $L^{2}$-stabilizability conditions for a class of nonstandard singularly perturbed functional-differential systems. Dyn. Contin. Discret. Impuls. Syst. Ser. B Appl. Algorithms 2009, 16, 181-213.

32. Ionita, A.; Dragan, V. Stabilization of singularly perturbed linear systems with delay and saturating control. In Proceedings of the 7th Mediterranean Conference on Control and Automation, Haifa, Israel, 28-30 June 1999; pp. 1855-1869.

33. Luse, D.W. Multivariable singularly perturbed feedback systems with time delay. IEEE Trans. Automat. Control 1987, 32, 990-994. [CrossRef]

34. Fridman, E. Effects of small delays on stability of singularly perturbed systems. Automat. J. IFAC 2002, 38, 897-902. [CrossRef]

35. Fridman, E. Stability of singularly perturbed differential-difference systems: An LMI approach. Dyn. Contin. Discret. Impuls. Syst. Ser. B Appl. Algorithms 2002, 9, 201-212.

36. Sun, F.; Zhou, L.; Zhang, Q.; Shen, Y. Stability bound analysis and synthesis for singularly perturbed systems with time-varying delay. Math. Probl. Eng. 2013, 2013, 517258. [CrossRef]

37. Gantmacher, F.R. The Theory of Matrices; Chelsea: New York, NY, USA, 1974; Volume 2. 